\title{
Toward Evaluation of Shape Memory Alloy Actuators for Endosurgery
}

\author{
Kaari Koehn and Shahram Payandeh \\ Experimental Robotics Laboratory (ERL) \\ School of Engineering Science, Simon Fraser University \\ Burnaby, B.C. V5A 1S6 \\ CANADA \\ kkoehn@sfu.cashahram@cs.sfu.ca
}

\begin{abstract}
Previous work in control of shape memory alloy actuators is examined and re-evaluated for possible application to design of tools for endosurgery. Flexible and rigid robotic links actuated by shape memory alloy are constructed and their behavior evaluated to determine and develop suitable control algorithms. The application of these links to electrically controlled surgical instruments such as graspers is discussed.
\end{abstract}

\section{INTRODUCTION}

Endosurgery, or minimally invasive surgery (MIS) is a dynamic field that has proven successful in reducing operation time and patient trauma. In endosurgery, access to the operation site is through a small incision or an existing orifice. This restricted access is a determining factor in instrument design. Two examples of this are laparoscopic gall bladder removal and endoscopic colon polyp removal. The instruments in laparoscopy are typically operated by mechanical linkages at the handles, which allow for graduated control and limited force feedback, but restrict the possible configurations and the working space of the tool at the surgical site. The tension and stiffness required in a passive endoscope restrict its turning radius. Manipulating the endoscope can also damage the soft surrounding tissue.

The research community has increasingly focused on investigating other sources of actuation for creating the movements of MIS tools. One such alternative could be the electromechanical shape memory alloy actuators. Inherent drawbacks and tradeoffs in giving up the mechanical linkage for an electromechanical one are the cost, complexity, and lack of tactile feedback. A poor user interface for the surgeon requires extra effort in mastering the techniques and performing the operation, and poses extra risk to the patient. However, if the interface problems of electromechanical instruments can be overcome, MIS will have more applications such as in teleoperation.

Nickel-Titanium (NiTi) alloy is a commercially available member of the family of Shape Memory Alloys (SMA). These alloys have two or three crystalline phases (martensite, austenite, and R-phase) and move between these phases when heated or mechanically stressed. The phase change results in a contraction when NiTi wire is heated. The force exerted by this contraction in comparison with the small diameter, light weight, and simplicity of operation makes NiTi worthy of consideration as an electrically-controlled micro-actuator. [1]
The heat- and stress-related phase changes affect the electrical resistance of the NiTi wire. This resistance change means the actuator can also serve as a feedback sensor. [2] These advantages of SMA microactuators have attracted attention from several researchers. One main disadvantage of SMA is the large time constant in natural cooling that makes the wire slow to relax. However, surface area/volume ratio increases as wire size decreases, and the disadvantage of slow cooling is less pronounced in the narrow wire of a microactuator. [2]

Other, more general disadvantages of SMA are energy efficiency and susceptibility to fatigue. [2] Applying SMA microactuators to minimally invasive surgery has its own set of challenges, such as heat dissipation within the body, current leakage, and conducivity to contamination and sterilization.

Previous works in SMA actuators are in active endoscopes $[3,4,5,6]$, piezoelectric-actuated rigid-link endoscopes [7], SMA-based tactile feedback [8,9], and endoscopic suturing [10]. Micro SMA control schemes can be based on the large amount of previous SMA work. $[11,12,13,14,2]$

This paper describes how two SMA-actuated links with accompanying control systems were developed. It starts with the computer simulations of the SMA actuator and continues through the preliminary experiments and positional control system for the flexible link before moving to position and force control in the rigid link and finishing with a discussion of endosurgical application and plans for future work.

\section{PRELIMINARY MODELLING}

As previously mentioned, much work has been done on modelling SMA in order to evaluate actuator performance. The mathematical models and control system simulations performed by Kuribayashi [11] were reproduced in the circuit simulator PSPICE and evaluated under different gains and actuator configurations. Kuribayashi examines counteracting-pair and spring-return SMA actuators in position- and force-feedback situations. Our positioncontrol simulation for counteracting SMA wires used the system of Figure 1(a) with parameters from Kuribayashi. The simulation under PSPICE differs from Kuribayashi's results, shown in Figure 2. PSPICE found the Laplace functions to be noncausal. PSPICE also states output as a voltage, which must be translated to appropriate units. We do not yet know why the results are different. Evaluation of the force simulation is likewise still in progress. 

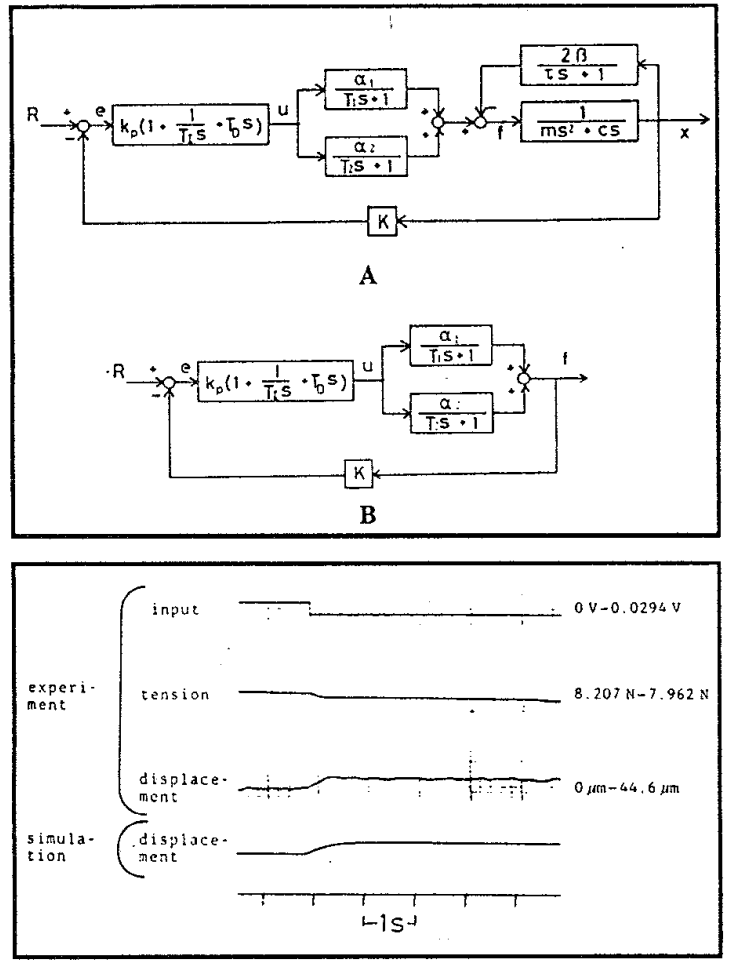

Figure 1: Mathematical modelling of antagonistic SMA actuator (from Kuribayashi) a) position control b) force control

Figure 2: Results of position simulation (from Kuribayashi)

\section{PRELIMINARY EXPERIMENTS}

To develop a more intuitive understanding of SMA behavior, a flexible link was constructed. The flexible link consists of $66 \mathrm{~cm}$. of $100 \mu \mathrm{m}$ NiTi wire doubled twice and glued to one side of a $14 \mathrm{~cm}$. strip of plastic acetate. Ikuta[2] refers to this doubling as an $\xi$-array, but it could also be termed an $\mathrm{N}$-array, where $\mathrm{N}$ refers to both the number of parallel wires (4, in this link) and to the zigzag pattern of the wires and connections.

At room temperature with still air, it was found that a given voltage of less than $9 \mathrm{v}$ applied to the flexible link will cause the link to curl into a predictable steady-state position. This behavior was qualitatively explained as follows: A voltage input $V$ will result in a power input to the SMA of $V^{2} R$ which is dissipated as heat. As the SMA temperature rises, the rate of heat output also rises, because the temperature differential with the surrounding air is higher. When the heat output matches the power input (in Watts), the wire will heat no further. This limit temperature, $T$, corresponds to a degree of phase transformation $\lambda[2]$ and thus a percentage contraction in the length of the SMA wire. The elastic force of the plastic strip resists the contraction, preventing the phase change from proceeding fully. The strain on the strip bends the flexible link's tip to some angle $\emptyset$ from the vertical.
Heating and cooling rates of SMA are dependent on the temperature and thermal conductivity of the surroundings and would likely vary inside and outside the body. [3] For this reason, a preliminary experiment was conducted that changed the ambient temperature to $10^{\circ} \mathrm{C}$ to observe the effects on the performance of the flexible link. Steady state voltage responses were obtained in a temperaturecontrolled chamber and are compared in Figure 4(c). The resistances for a given voltage were higher at the lower temperature.

\section{FLEXIBLE LINK MODELLING AND CONTROL}

Modelling and control was done with an AT-MIO-L16 interface card and LabWindows/CVI software libraries from National Instruments. A simple interface circuit was simulated in PSPICE and constructed so the high DC current demands of the SMA could be met without damaging the interface card.

The flexible link control range was set as $5^{\circ}$ to $25^{\circ}$ from the fully straightened vertical position to avoid excessively long cooling times and thermal runaway. Observed angles were recorded at the top of the acetate strip.

\section{Open-Loop Position Control}

The open-loop control system shown in Figure 3(a) used the steady-state voltage-position curve fit seen in Figure 4(b) as a lookup table.

\section{Feedforward}

Due to the slow response of the system in reaching an input position, feedforward control was added to reduce response time in both heating and cooling. The 'disturbance' is the difference between the final position and the known start position $\emptyset_{0}$. The chosen feedforward scheme applied the voltage corresponding to a position $150 \%$ further than the actual final position. This voltage was maintained for a period of time depending on the final position, the size of the step change, and the direction of the step (heating or cooling). Times were determined experimentally in 6 cases then approximated with the following formula:

$$
\mathrm{t}=\left(85 /\left(\emptyset_{f}^{2} / 5+\emptyset_{f}-\emptyset_{\mathrm{o}}\right)\right)^{1.5},
$$

where $t$ is hold time in seconds, $\emptyset_{o}$ is start position and $\emptyset_{f}$ is final position.

This feedforward control resulted in significant speed improvements. For example, a position change from $15^{\circ}$ to $10^{\circ}(\sim 6 \mathrm{v}$ to $\sim 4 \mathrm{v})$ took $40 \mathrm{~s}$ to come within $1^{\circ}$ of final position under natural cooling, and 4 s to achieve the same step using feedforward, which temporarily reduced the voltage to $0 \mathrm{v}$ for maximum cooling. Figure 5 compares this feedforward control with open-loop and closed-loop in a step from $10^{\circ}$ to $15^{\circ}$. 


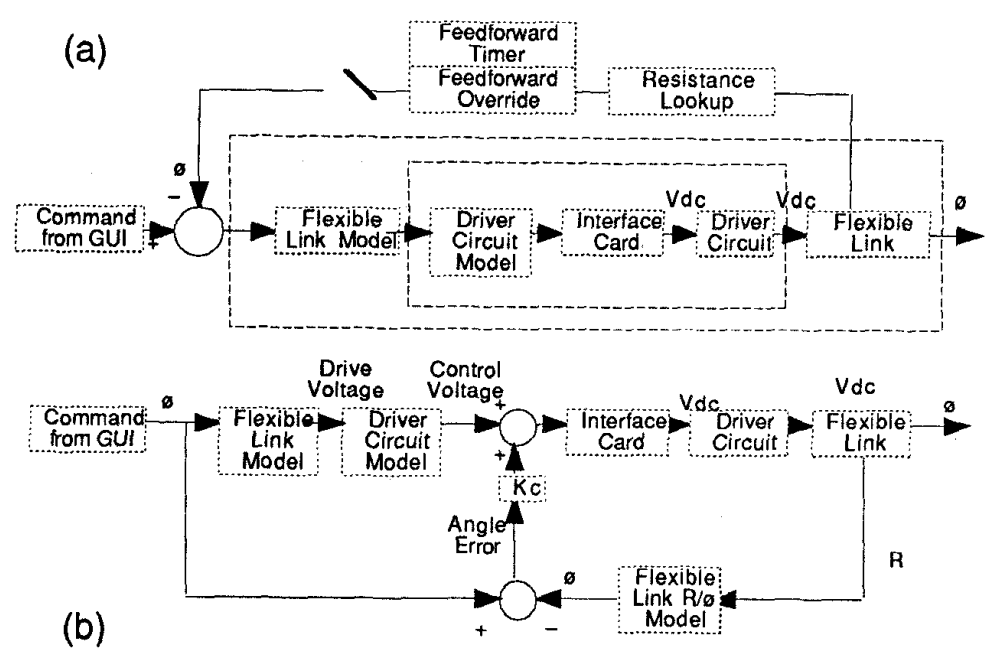

Figure 3: Flexible link control a) open-loop (optional feedforward) b) closed-loop

\section{Closed-Loop Proportional Position Control}

Figure 3(b) shows the closed-loop control system for the flexible link that varied control voltage proportionally with position error. Closed loop control used the SMA resistance-position lookup in Figure 4 to determine changes to the control voltage. The control voltage was originally incremented or decremented by a fixed amount corresponding to $\sim 0.1^{\circ}$, but this amount is now multiplied by the magnitude of the position error for faster response. Figure 5 compares the open-loop control to proportional closed-loop error correction (and feedforward) for the same reference input.

Error at the high end of the resistance/position lookup table led to an unstable system when the proportional closedloop control was combined with feedforward. The voltage increased until the experiment was halted to avoid damaging the link. This error is a function of the starting temperature of the wire, among other factors, and a more complex model would be needed to avoid it.

\section{RIGID LINK MODELLING AND CONTROL}

The rigid link has a $7.62 \mathrm{~cm}$ metal arm and is actuated by $15 \mathrm{~cm}$ of $100 \mu \mathrm{m}$ SMA attached to a plastic cylinder of $0.8 \mathrm{~cm}$ radius mounted coaxially. The experimental setup and interface circuit were slightly modified from those used for the flexible link.

\section{Open-loop Experimentation}

Step-response experimentation showed that the $5 \mathrm{~g}$ weighted rigid link had a large change in steady-state positions over a small voltage range, as seen in Figure 6. This can be related to the increase in stress as seen by Stoeckel and Simpson [1] when rising temperature reaches the first 'martensic yield point'. The link will always undergo this position change over a narrow voltage (temperature) range when the return force is a constant load or weight. Therefore, the type of open-loop control used in the flexible link is only applicable when the resistive force varies with position, like a spring. The yield point temperature increases with resistive force.

\section{Closed-loop Control}

For closed-loop control, three joint angle feedback methods were evaluated: SMA resistance, a potentiometer mounted on the axis, and a LED-mirror-CdS photocell arrangement with the mirror mounted on the axis.

Data from each angle sensor was collected along with observed angles, and then an appropriate curve was fitted to the data to allow angle lookup.

The SMA resistance was able to determine the angle within $10^{\circ}$ during a contraction stage, as seen in Figure 7(a), but could not use the same lookup table to predict angle during a relaxation stage due to hysteresis. It was also unable to predict position when the link was disturbed by external forces.

The potentiometer lookup table is shown in Figure 7(b). The angle error was as much as $15^{\circ}$ due to backlash in the mechanical link construction. The variation was much less if the direction of motion was known, because backlash could be accounted for. The friction of the potentiometer contact is an inherent disadvantage in this feedback method because it introduces an additional disturbance force to the mechanical system.

The light-based angle sensor (LBAS) was constructed to avoid this friction problem. Two shielded CdS photocells receive the light from an LED that shines on the axiallymounted mirror. Changes in the rigid link angle affect the light reaching the photocells and can be seen as a change in the resistance of the cells. Figure 7(c) shows the lookup table using the logarithm of the difference in photocell resistances. In this approach, angle error was within $5^{\circ}$.

The ability of such a sensor to be miniaturized was considered. It would require micromachined laser diodes, photoresistors or phototransistors, and mirrored surfaces on the end of the actuator arm. As such, it would seem to be feasible to manufacture. 

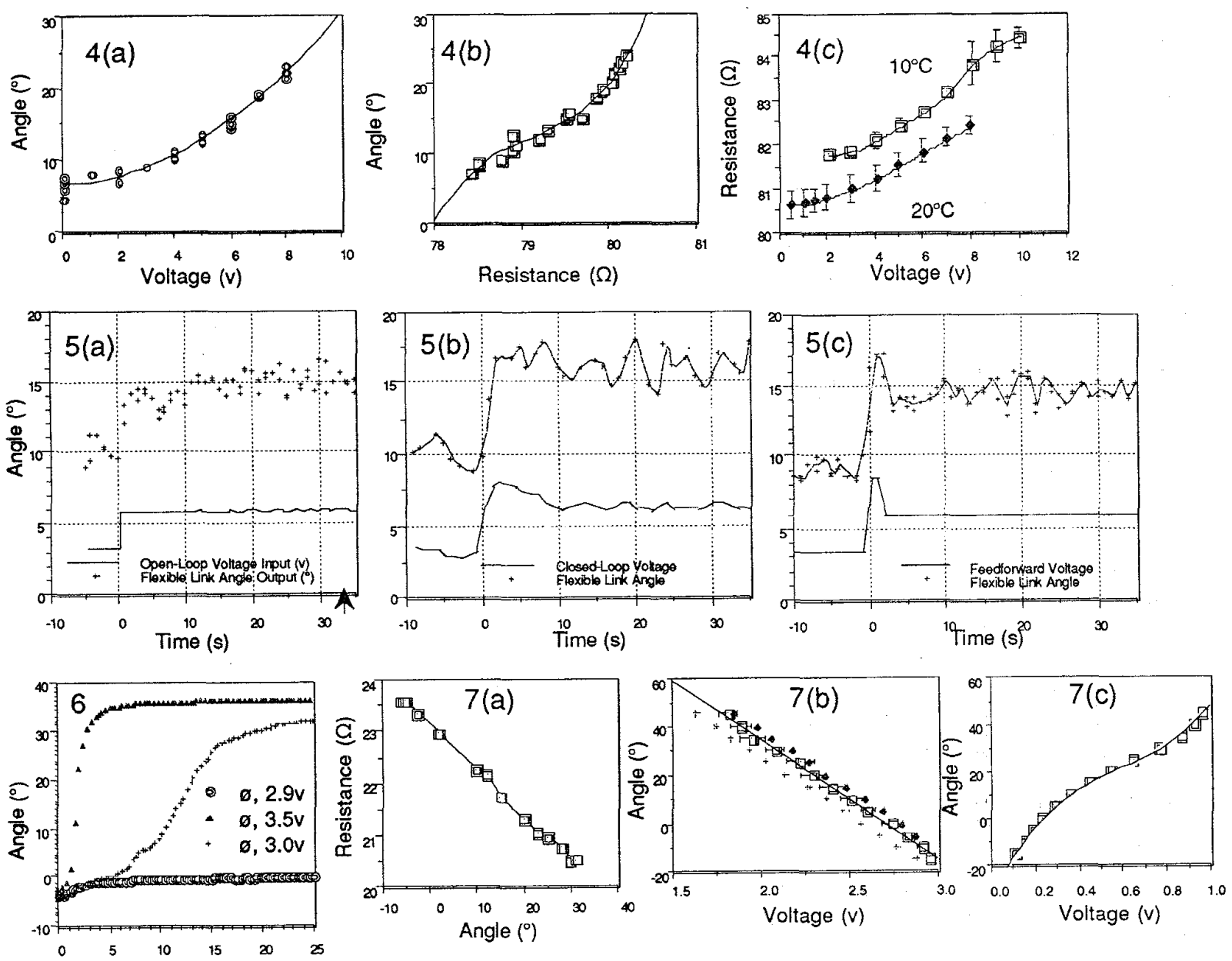

Time (s)

Fig. 4: Flexible link steady states a) position/voltage b) position/resistance c) resistance/voltage

Fig. 5: Flexible link step response a) open-loop b) closed-loop c) feedforward

Fig. 6: Rigid link open-loop step responses (for 3 step sizes)

Fig. 7: Rigid link angle feedback lookup tables a) SMA resistance during contraction cycle b) Potentiometer, showing backlash c) LED/CdS sensor voltages

The chosen control scheme was to apply an overvoltage until the feedback sensor reported the angle had been reached. A hold voltage was then applied to prevent the SMA from relaxing. The hold voltage was dependent on the final angle and not the direction or magnitude of the step. Figure 8 shows the early results of this control method for a positive (contraction) and negative (relaxation) angle change, using all three feedback methods in turn. It can be seen that the SMA resistance feedback gives good control on a contraction but poor control in a relaxation.

The proportional position control from the flexible link did not work with the link, as the steep slope of the voltage response (as seen in Figure 6) kept such a system oscillating more than $10^{\circ}$ about the desired position of $15^{\circ}$. A PID controller might serve better.

\section{Force Control}

Because the rigid link has the possibility to be used as a gripper or forceps, it was necessary to determine if the exerted force of the rigid link was suitable for gripping. The experimental setup mounted a bracket at $2.5 \mathrm{~cm}$ from the axis of the rigid link. A force-sensing resistor pad was taped to the bracket at the contact point to measure the force exerted on the bracket by the rigid link.

The results of this experiment indicate that the amount of force exerted on the FSR varies with the voltage applied to the SMA as per Figure 9. The FSR was calibrated with weights using approximately the same contact area, and these points are marked on the graph, showing that the rigid link was exerting nearly $1 \mathrm{~N}$ of force at $2.5 \mathrm{~cm}$ from the axis at $4.8 \mathrm{~V}$. (The force would be $1 / 3$ of this at the end of the arm, due to reduced torque.) This force can be increased by using an $\mathrm{N}$-array (more wires in parallel). 

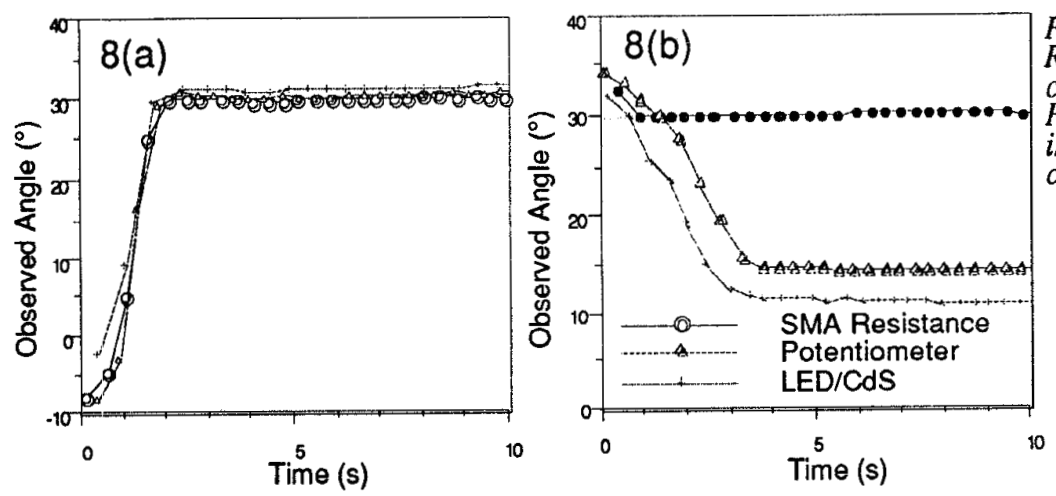

Fig. 8: Rigid Link Closed-Loop Step Response under 3 feedback methods a) contraction, $-5^{\circ}$ to $30^{\circ}$ b) relaxation, $33^{\circ}$ to 15

Fig. 9: Rigid Link Force Tests a) varying input over time b) SMA resistance from (a) c) force output from (a)
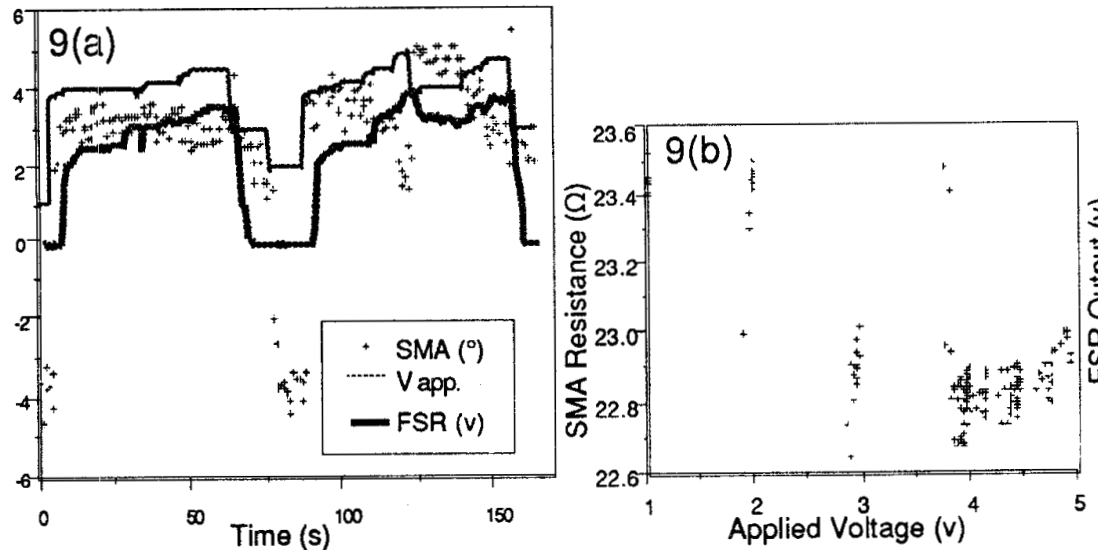

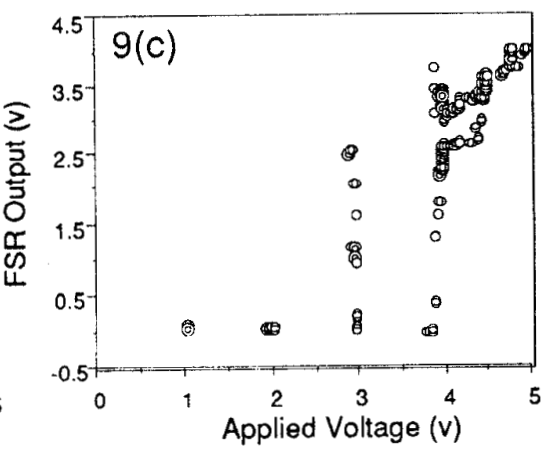

The upwards curve near 5v in Figure 9(b) shows the SMA resistance change restricted by stress. The angle calculated from the SMA resistance varies more widely from the angle reported by the angle sensor when the arm is blocked at $10^{\circ}$ and force is being exerted. The rigid link in combination with an angle sensor can thus provide both contact force and angle feedback.

\section{DISCUSSION}

The microactive catheter of Ikuta [5] and the locking endoscope of Sturges [4] both use the same principles as the flexible link of Section 3. Several flexible links joined at the ends allow positional control without exerting a great deal of force on a surrounding channel, which is a benefit for an endoscope attempting to navigate a turn in the intestinal tract without damaging the walls.

Snake motion and the slide motion scheme proposed by Sturges [4] are also possible with SMA actuators. It may be worthwhile to look at the locomotion of centipedes and earthworms for locomotion strategies in a softly resisting enviroment. For example, an expanding bellows might aid a slide-motion endoscope from losing ground as it contracts longitudinally.

The rigid link can use even gross positional control to act as a forceps and clamp onto an object to be steadied or retrieved. Finer control would allow it to follow more closely the actions of a human surgeon, say, and this control is apparently possible.
Surgical tasks such as suturing can be automated through ingenious mechanical design. [15] The SMA actuators should be a basic part of a designer's toolkit.

SMA actuators can be miniaturized using IC manufacturing techniques to create thin-film SMA depositions. $[2,16]$ To prevent current leakage during surgery, SMA actuators might be given a flexible insulative coating. Such a coating should be heat-conductive to aid SMA cooling. A coating that fills in small gaps and pits would aid sterilization of the SMA-actuated instrument.

\section{CONCLUSIONS / FUTURE WORK}

The control schemes proposed here are simple and have not been checked for stability. Implementation of a digital PID controller [17] is in progress.

The discrepancy between Kuribayashi's simulation results, and my results from attempting to run the same simulation will be investigated further. One clue is the simulator's report of a non-causal system.

Future work will implement $\mathrm{N}$-arrays of parallel wire on the rigid link for exerting more force. The rigid link will also be tested with antagonistic SMA to supply resistive force.

A thermocouple as suggested by Kuribayashi [12] may help force feedback by reporting the SMA temperature and thus the expected SMA phase change. 
A micromachined or millimeter-scale rigid link may be attempted. The miniature link can then be tested for current leakage and temperature effects under more realisic surgical conditions. The final evaluation for endosurgery will compare the performance of a microgripper using the rigid link to the demands of endosurgical tasks.

\section{REFERENCES}

[1] Stoeckel, D. and Simpson, J. 1992. "Actuation and control with Ni-Ti shape memory alloys", Active Materials and Adaptive Structures. Proc. of the ADPA/AIAA/ASME/SPIE Conference 157-160.

[2] Ikuta, K. 1990. "Micro/Miniature Shape Memory Alloy Actuator", Proc.IEEE Int. Conf. Robotics and Automation 21562161.

[3] Fukuda, T., Guo, S., Kosuge, K., Arai, F., Negoro, M., and Nakabayashi, K. May 1994. "Micro Active Catheter System with Multi Degrees of Freedom", Proc. 1994 IEEE Int.Conf. on Robotics and Automation Vol.3, 2290-2295.

[4] Sturges, R.H. and Laowattana, S. April 1993. "A Flexible, Tendon-Controlled Device for Endoscopy", The International Journal of Robotics Research Vol.12, 121-131.

[5] Ikuta, K., Tsukamoto, M., and Hirose, S. 1988. "Shape Memory Alloy Servo Actuator System With Electric Resistance Feedback And Application For Active Endoscope", Proc. IEEE Int. Conf. Robotics and Automation 427-430.

[6] Kaplan, H. February 1995. "Steering Borescopes With a Shape-Memory Alloy", Photonics Spectra 44-46.

[7] Ikuta, K., Nokata, M., and Aritomi, S. 1994. "Biomedical Micro Robots Driven By Miniature Cybernetic Actuator", Proc. IEEE Micro Electrical Mechanical Systems 263-268.

[8] Hasser, C. J. and Weisenberger, J. M. 1993. "Preliminary evaluation of a shape-memory alloy tactile feedback display", Advances in Robotics, Mechatronics, and Haptic Interfaces ASME DSC-Vol.49, 73-79.

[9] Kontarinis, D. A. and Howe, R. D. 1993. "Tactile Display of Contact Shape in Dextrous Telemanipulation", Advances in Robotics, Mechatronics, and Haptic Interfaces ASME DSCVol.49, 81-88.

[10] Melzer, A., Schurr, M.O., Lirici, M.M., Klemm, B., Stöckel, D., and Buess, G. 1994. "Future Trends in Endoscopic Suturing", Endoscopic Surgery and Allied Technologies 78-82.

[11] Kuribayashi, K. Winter 1986. "A New Actuator of a Joint Mechanism Using TiNi Alloy Wire", The International Journal of Robotics Research Vol.4 No.4, 47-58.

[12] Kuribayashi, K. February 1991. "Improvement of the Response of an SMA Actuator Using a Temperature Sensor", The International Journal of Robotics Research Vol.10 No.1, 13-20.

[13] Lin, G., Yang, D., and Warrington, R.O, 1992. "A Computational Model of the Shape Memory Alloys for the Design and Control of Micro Actuators", Micromechanical Systems ASME DSC-Vol.40, 171-181.
[14] Madill, D.R. and Wang, D. May 1994. "The Modelling and L2-Stability of a Shape Memory Alloy Position Control System", Proceedings 1994 IEEE International Conference on Robotics and Automation Vol.1, 293-299.

[15] Nagy, A. and Payandeh, S. 1994. "Endoscopic EndEffectors", ASME National Design Engineering Conference, Chicago, Illinois, paper \#94-DE-5.

[16] Johnson, A.D. March 1991. "Vacuum-deposited TiNi shape memory film: characterization and applications in microdevices", Journal of Micromechanics and Microengineering Vol.1 \#1 3441.

[17] Phillips, Charles L. and Harbor, Royce D. 1991. Feedback Control Systems 2nd ed. Prentice Hall, Eaglewood Cliffs, New Jersey. 